\title{
Napyradiomycin A1, an inhibitor of mitochondrial complexes I and II
}

\author{
Kohta Yamamoto ${ }^{1}$, Etsu Tashiro ${ }^{1}$, Keiichiro Motohashi ${ }^{2}$, Haruo Seto ${ }^{2}$ and Masaya Imoto ${ }^{1}$ \\ The Journal of Antibiotics (2012) 65, 211-214; doi:10.1038/ja.2011.138; published online 18 January 2012
}

Keywords: mitochondrial electron transport; napyradiomycin; intracellular ATP level

We have previously proposed a cell-based screening method 'EGFinduced (epidermal growth factor) filopodium protrusion assay' to identify mitochondrial electron transport inhibitors or glycolysis inhibitors. Filopodia are spike-like cell membrane projections that contribute to tumor metastasis. Previously, we have reported that mitochondrial electron transport inhibition resulted in the inhibition of EGF-induced filopodium protrusion in human adenocarcinoma A431 cells only when their glycolytic pathways were restricted. ${ }^{1}$ By using the inhibition of filopodium protrusion as an indicator, we identified napyradiomycin A1 (Figure 1a; isolated from Streptomyces antimycoticus NT17), ${ }^{2}$ which was previously identified as an antibacterial antibiotic, ${ }^{3}$ as a candidate of mitochondrial electron transport inhibitor. A431 cells were treated with napyradiomycin A1 with or without $10 \mathrm{~mm}$ 2-deoxy-D-glucose (Sigma-Aldrich, St Louis, MO, USA) for $30 \mathrm{~min}$, followed by $30 \mathrm{ng} \mathrm{m}^{-1}$ EGF (Sigma-Aldrich) stimulation and observation under a microscope. As shown in Figure 1b, $20 \mu \mathrm{m}$ napyradiomycin Al inhibited EGF-induced filopodium protrusion in A431 cells only in the presence of the glycolytic enzyme hexokinase inhibitor 2-deoxy-D-glucose. Mitochondrial electron transport inhibitor rotenone (Sigma-Aldrich) also inhibited filopodium protrusion only in the presence of 2-deoxy-D-glucose. Furthermore, it was reported that co-treatment with a mitochondrial electron transport inhibitor and a glycolytic inhibitor markedly decreased intracellular ATP levels. ${ }^{1}$ We then tested whether napyradiomycin A1 decreased ATP levels in A431 cells in which glycolytic pathways were restricted. As intracellular ATP levels were not affected by EGF stimulation (data not shown), we measured intracellular ATP levels under the condition where A431 cells were treated with napyradiomycin $\mathrm{A} 1$ with or without $10 \mathrm{~mm}$ 2-deoxy-D-glucose for $30 \mathrm{~min}$ in the absence of EGF. After incubation, intracellular ATP levels were measured using a Cell Titer-Glo Luminescent Cell Viability Assay Kit (Promega, Madison, WI, USA) with a luminometer (Wallac; Perkin-Elmer, Waltham, MA, USA). As shown in Figure 1c, napyradiomycin A1 treatment did not decrease cellular ATP levels; however,
$20 \mu \mathrm{m}$ napyradiomycin A1 markedly decreased cellular ATP levels in the presence of 2-deoxy-D-glucose in A431 cells. Furthermore, ATP levels in HeLa cells also decreased under co-treatment with napyradiomycin A1 and 2-deoxy-D-glucose (data not shown). These results suggested that napyradiomycin A1 inhibited mitochondrial electron transport in cancer cells.

Next, we examined whether napyradiomycin Al actually inhibited mitochondrial electron transport in vitro by using submitochondrial particles (SMP) obtained from the bovine heart. In order to prepare SMP, bovine hearts were homogenized in MSH buffer $(210 \mathrm{~mm}$ mannitol, $70 \mathrm{~mm}$ sucrose, $1 \mathrm{~mm}$ DTT, $1 \mathrm{~mm}$ EGTA, $0.1 \%$ BSA and $10 \mathrm{~mm}$ HEPES pH 7.4) with a Potter-Elvehjem homogenizer (Nippon genetics, Tokyo, Japan). Homogenates were centrifuged at $1000 \mathrm{~g}$ for $10 \mathrm{~min}$, and the resulting supernatant was further centrifuged at $8000 \mathrm{~g}$ for $20 \mathrm{~min}$. Pellets were suspended in MSH buffer and obtained as SMP. ${ }^{4}$

The mitochondrial electron transport chain consists of four discrete multisubunit complexes: NADH-ubiquinone oxidoreductase (complex I), succinate-ubiquinone oxidoreductase (complex II), ubiquinol-cytochrome $c$ oxidoreductase (complex III) and cytochrome $c$ oxidase (complex IV). Therefore, we evaluated which complex was the target of napyradiomycin Al.

Mitochondrial complex I activity was measured by monitoring the absorbance change of NADH at $340 \mathrm{~nm}$ in the presence of antimycin A (Sigma-Aldrich) and KCN (Sigma-Aldrich), an inhibitor of complex III and complex IV, respectively. ${ }^{5,6}$ The enzyme assay was performed at $30^{\circ} \mathrm{C}$ in a buffer containing $50 \mathrm{~mm}$ phosphate ( $\mathrm{pH} 7.4), 250 \mathrm{~mm}$ sucrose, $0.1 \mu \mathrm{g} \mathrm{ml}^{-1}$ antimycin A, $2 \mathrm{~mm} \mathrm{KCN}$, $50 \mu \mathrm{m}$ decylubiquinone (DB; 2,3-dimethoxy-5-methyl-6-decyl-1,4benzoquinone, an exogenous hydrophobic quinone that acts as electron acceptor; Sigma-Aldrich), ${ }^{7} 0.2 \mathrm{~mm} \mathrm{NADH}$, and $12 \mu \mathrm{g} \mathrm{ml}^{-1}$ SMP with or without napyradiomycin A1. Rotenone was used as a positive control of complex I inhibitor. We found that napyradiomycin A1 inhibited complex I activity with an $\mathrm{IC}_{50}$ value of $20 \mu \mathrm{m}$ (Figure 2a).

${ }^{1}$ Chemical Biology Laboratory, Department of Biosciences and Informatics, Faculty of Science and Technology, Keio University, Yokohama City, Japan and ${ }^{2}$ Bioregulatory Laboratory, Faculty of Applied Biosciences, Tokyo University of Agriculture, Tokyo, Japan

Correspondence: Dr E Tashiro, Department of Biosciences and Informatics, Faculty of Science and Technology, Chemical Biology Laboratory, Faculty of Science and Technology, Keio University, 3-14-1 Hiyoshi, Kohoku-ku, Yokohama 223-8522, Japan.

E-mail: tashiro@bio.keio.ac.jp

Received 2 December 2011; revised 15 December 2011; accepted 16 December 2011; published online 18 January 2012 
<smiles>CC(C)=CCC/C(C)=C/C[C@@]12OC(C)(C)[C@@H](Cl)CC1(Cl)C(=O)c1c(O)cc(O)cc1C2=O</smiles>
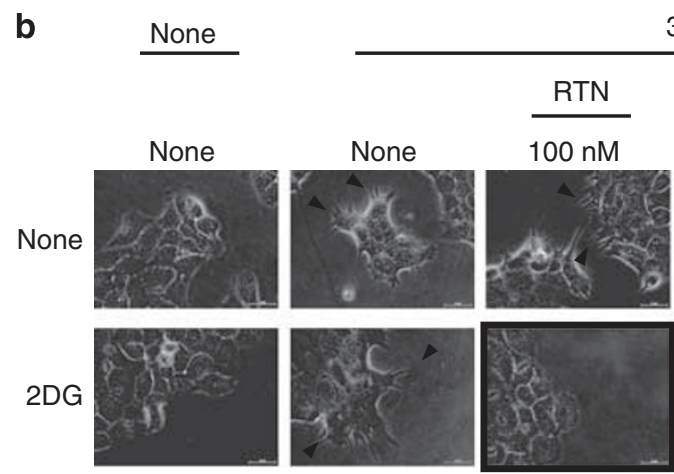

$30 \mathrm{ng} / \mathrm{ml}$ EGF

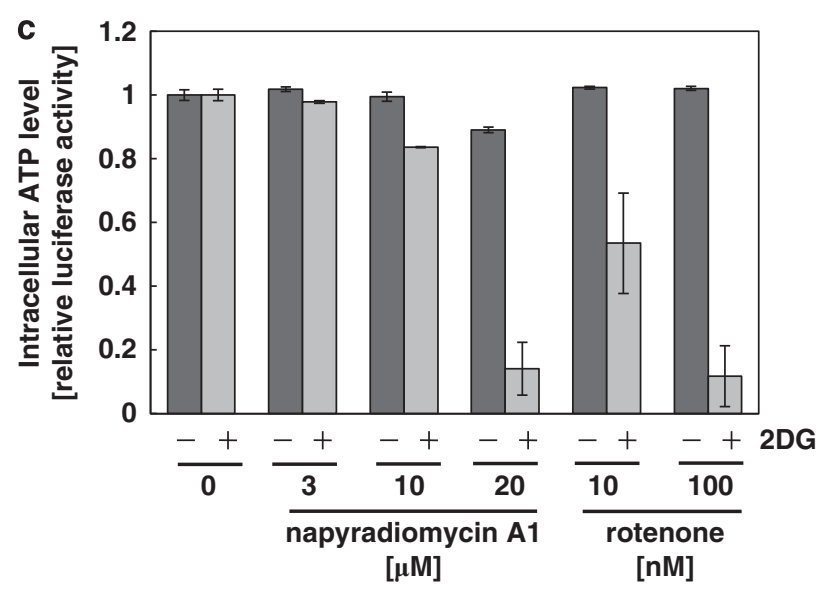

Figure 1 Napyradiomycin A1 decreased intracellular ATP levels. (a) The structure of napyradiomycin A1. ${ }^{3}$ (b) Napyradiomycin A1 inhibited EGF-induced filopodia protrusion only in the presence of 2 -deoxy-D-glucose (2DG, $10 \mathrm{~mm}$ ). The assay method was as described previously. ${ }^{1}$ Mitochondrial electron transport inhibitor rotenone (RTN $100 \mathrm{~nm}$ ) was used as a positive control. Arrowhead indicates filopodia, and the framed photos show filopodium inhibition. (c) Napyradiomycin A1 decreased intracellular ATP levels in 2DG-treated A431 cells. A431 cells were treated with napyradiomycin A1 with or without 10 mm of 2DG for $30 \mathrm{~min}$. After incubation, intracellular ATP levels were measured using a Cell Titer-Glo Luminescent Cell Viability Assay Kit with a luminometer.

Mitochondrial complex II activity was measured by monitoring the absorbance change of 2,6-dichlorophenolindophenol (Sigma-Aldrich) at $600 \mathrm{~nm}$ in the presence of rotenone and $\mathrm{KCN}^{8}{ }^{8}$ The enzyme assay was performed at $30^{\circ} \mathrm{C}$ in a buffer containing $50 \mathrm{~mm}$ phosphate $(\mathrm{pH}$ 7.4), $0.1 \mu \mathrm{m}$ rotenone, $2 \mathrm{~mm} \mathrm{KCN}, 40 \mu \mathrm{m}$ 2,6-dichlorophenolindophenol, and $12 \mu \mathrm{g} \mathrm{ml}^{-1} \mathrm{SMP}$ with or without napyradiomycin A1. Theonoyl trifluoroacetone (Sigma-Aldrich) was used as a positive control of complex II inhibitor. ${ }^{9}$ We found that napyradiomycin A1 inhibited complex II activity with an $\mathrm{IC}_{50}$ value of $9.7 \mu \mathrm{M}$ (Figure $2 \mathrm{~b}$ ).

Mitochondrial complex III activity was measured by monitoring the absorbance change of the reduction of oxidized cytochrome $c$ at $550 \mathrm{~nm}$ in the presence of rotenone, $\mathrm{KCN}$ and an electron donor decylubiquinol. Decylubiquinol was obtained by the reduction of DB with sodium borohydride. ${ }^{10}$ The enzyme assay was performed at $30^{\circ} \mathrm{C}$ in a buffer containing $50 \mathrm{~mm}$ Tris (pH 7.6), $1 \mathrm{mM} \mathrm{MgCl}_{2}, 0.1 \mu \mathrm{M}$ rotenone, $2 \mathrm{~mm} \mathrm{KCN}, 40 \mu \mathrm{M}$ cytochrome $c, 50 \mu \mathrm{M}$ decylubiquinol and $12 \mu \mathrm{g} \mathrm{ml}^{-1} \mathrm{SMP}$ with or without napyradiomycin A1. Although antimycin A inhibited complex III activity, napyradiomycin Al did not, even at $20 \mu \mathrm{M}$ (Figure 2c)

Mitochondrial complex IV activity was measured by monitoring the absorbance change of the oxidation of reduced cytochrome $c$ at $550 \mathrm{~nm}$. The enzyme assay was performed at $30^{\circ} \mathrm{C}$ in a buffer containing $50 \mathrm{~mm}$ Tris ( $\mathrm{pH} 7.6$ ), $1 \mathrm{~mm} \mathrm{MgCl}_{2}, 0.1 \mu \mathrm{g} \mathrm{ml}^{-1}$ antimycin A, $0.1 \mu \mathrm{M}$ rotenone, $20 \mu \mathrm{M}$ reduced cytochrome $c$ and $12 \mu \mathrm{g} \mathrm{ml}^{-1} \mathrm{SMP}$ 
a
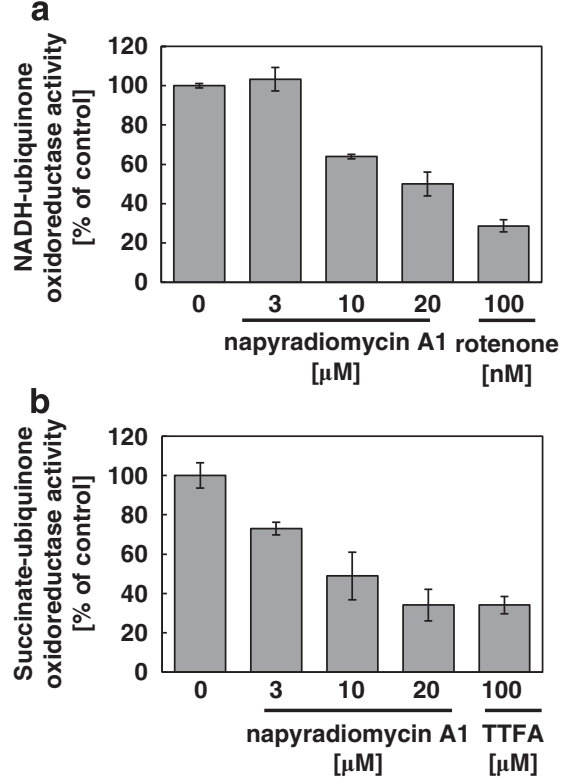

C

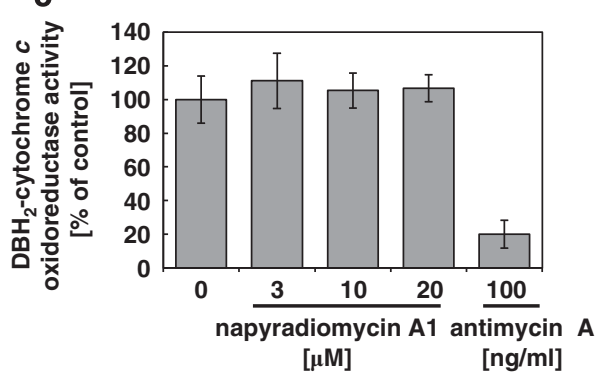

d

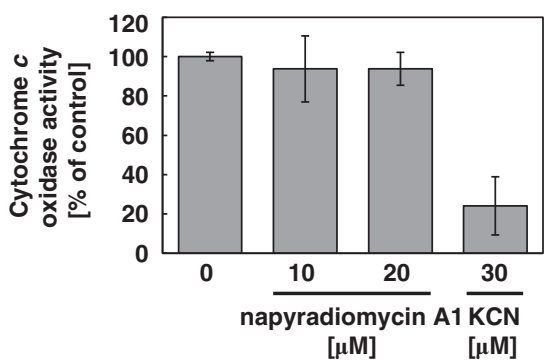

Figure 2 Napyradiomycin A1 inhibited mitochondrial complexes I and II. A modification of previously reported methods ${ }^{10,16-18}$ was used to measure complex I, complex II, complex III and complex IV activities. (a) Napyradiomycin Al inhibited complex I activity. Mitochondrial complex I (NADHubiquinone oxidoreductase) activity was measured by monitoring the absorbance change of NADH at $340 \mathrm{~nm}$ using SMP. (b) Napyradiomycin A1 inhibited complex II activity. Mitochondrial complex II (Succinate-ubiquinone oxidoreductase) activity was measured by monitoring the absorbance change of 2,6dichlorophenolindophenol at $600 \mathrm{~nm}$ using SMP. (c) Napyradiomycin A1 did not inhibit complex III activity. Mitochondrial complex III (decylubiquinol $\left(\mathrm{DBH}_{2}\right)$-cytochrome $c$ oxidoreductase) activity was measured by monitoring the absorbance change of cytochrome $c$ at $550 \mathrm{~nm}$ using SMP. (d) Napyradiomycin A1 did not inhibit complex IV activity. Mitochondrial complex IV (cytochrome $c$ oxidase) activity was measured by monitoring the absorbance change of the oxidation of reduced cytochrome $c$ at $550 \mathrm{~nm}$ using SMP. SMP were obtained by standard different centrifugation. ${ }^{4}$ All data are the mean \pm s.d. of three independent experiments.

with or without napyradiomycin A1. Although $\mathrm{KCN}$ inhibited complex IV activity, $20 \mu \mathrm{M}$ napyradiomycin A1 inhibited complex IV activity less than $10 \%$ (Figure $2 \mathrm{~d}$ ).

In conclusion, napyradiomycin A1 inhibited mitochondrial complexes I and II, but did not inhibit complex III or IV in vitro. Therefore, it was suggested that mitochondrial electron transport inhibition by napyradiomycin A1 was caused by the inhibition of mitochondrial complexes I and II.

It was reported that the isoprenyl unit of ubiquinone was important to bind to mitochondrial complex $\mathrm{I}^{11}$ It has been also reported that two isoprene units of ubiquinone could be modeled in the native structure of complex II by computational analysis using a proteinligand docking program. ${ }^{12}$ Napyradiomycin A1 is structurally similar to ubiquinone (Figure 1a); therefore, it is likely that napyradiomycin A1 binds with the ubiquinone-binding site of complexes I and II through its terpenoid residue. On the other hand, although mitochondrial complex III also includes a ubiquinone-binding site, ${ }^{13}$ napyradiomycin A1 was unable to inhibit the activity of complex III up to $20 \mu \mathrm{M}$. Our result that napyradiomycin A1 inhibits mitochondrial complexes I and II but not complex III is interesting, because it has been reported that the structure of ubiquinone-binding sites in complex I and complex III may be similar, but they are different from a ubiquinone-binding site in complex II. ${ }^{14}$ Further study is necessary to elucidate the mechanism by which napyradiomycin Al inhibits mitochondrial complexes I and II but not complex III.

Isolation of napyradiomycins was first reported in 1986. Napyradiomycin group compounds were isolated from Actinomycetes and identified as antibacterial antibiotics. ${ }^{3}$ At present, many napyradiomycins have been identified and reported to exhibit cytotoxicity against human cancer cell lines; ${ }^{15}$ however, the molecular mechanism by which napyradiomycins show cytotoxicity has been unclear. Our results raise the possibility that an inhibitory effect of napyradiomycin Al against the mitochondrial electron transport chain may explain the cytotoxicity of napyradiomycins against cancer cells.

\section{ACKNOWLEDGEMENTS}

This study was partly supported by a Grant-in-Aid for Scientific Research from the Ministry of Education, Culture, Sports, Science and Technology of Japan. This study was also supported by grants from Suzuken Memorial Foundation. This study was supported in part by the Global COE Program for Human Metabolomic Systems Biology of MEXT, Japan.

1 Kitagawa, M., Ikeda, S., Tashiro, E., Soga, T. \& Imoto, M. Metabolomic identification of the target of the filopodia protrusion inhibitor glucopiericidin A. Chem. Biol. 17, 989-998 (2010)

2 Motohashi, K., Sue, M., Furihata, K., Ito, S. \& Seto, H. Terpenoids Produced by Actinomycetes: Napyradiomycins from Streptomyces antimycoticus NT17. J. Nat. Prod. 71, 595-601 (2008)

3 Shiomi, K. et al. Novel antibiotics napyradiomycins. Production, isolation, physicochemical properties and biological activity. J. Antibiot. 39, 487-493 (1986).

4 Pedersen, P L. et al. Preparation and characterization of mitochondria and submitochondrial particles of rat liver and liver-derived tissues. Methods Cell Biol. 20, 411-481 (1978).

5 di Rago, J P. \& Colson, A M. Molecular basis for resistance to antimycin and diuron, $Q$-cycle inhibitors acting at the $Q_{\mathrm{i}}$ site in the mitochondrial ubiquinol-cytochrome $\mathrm{C}$ reductase in Saccharomyces cerevisiae. J. Biol. Chem. 263, 12564-12570 (1988).

6 Chance, B. The kinetics and inhibition of cytochrome components of the succinic oxidase system. III. Cytochrome b. J. Biol. Chem. 233, 1223-1229 (1958).

7 Lenaz, G. et al. Coenzyme $Q$ deficiency in mitochondria: kinetic saturation versus physical saturation. Mol. Aspects Med. 18(Suppl), S25-31 (1997).

8 Chance, B., Williams, G R. \& Hollunger, G. Inhibition of electron and energy transfer in mitochondria. I. Effects of Amytal, thiopental, rotenone, progesterone, and methylene glycol. J. Biol. Chem. 238, 418-431 (1963). 
9 Paddenberg, R. et al. Essential role of complex II of the respiratory chain in hypoxiainduced ROS generation in the pulmonary vasculature. Am. J. Physiol. Lung Cell Mol. Physiol. 284, L710-719 (2003).

10 Rhein, V. et al. Amyloid-beta leads to impaired cellular respiration, energy production and mitochondrial electron chain complex activities in human neuroblastoma cells. Cell Mol. Neurobiol. 29, 1063-1071 (2009).

11 Warncke, K. et al. Influence of hydrocarbon tail structure on quinone binding and electron-transfer performance at the $Q_{A}$ and $Q_{B}$ sites of the photosynthetic reaction center protein. Biochemistry 33, 7830-7841 (1994).

12 Horsefield, R. et al. Structural and computational analysis of the quinone-binding site of complex II (succinate-ubiquinone oxidoreductase): a mechanism of electron transfer and proton conduction during ubiquinone reduction. J. Biol. Chem. 281, 7309-7316 (2006).

$13 \mathrm{Xia}$, D. et al. Crystal structure of the cytochrome $\mathrm{bc}_{1}$ complex from bovine heart mitochondria. Science 277, 60-66 (1997).
14 Tan, A. K., Ramsay, R. R., Singer, T. P. \& Miyoshi, H. Comparison of the structures of the quinone-binding sites in beef heart mitochondria. J. Biol. Chem. 268, 19328-19333 (1993).

15 Soria-Mercado, I. E., Prieto-Davo, A., Jensen, P. R. \& Fenical, W. Antibiotic terpenoid chloro-dihydroquinones from a new marine actinomycete. J. Nat. Prod. 68, 904-910 (2005).

16 Brusque, A. M. et al. Inhibition of the mitochondrial respiratory chain complex activities in rat cerebral cortex by methylmalonic acid. Neurochem. Int. 40, 593-601 (2002).

17 Telford, J. E., Kilbride, S. M. \& Davey, G. P. Decylubiquinone increases mitochondrial function in synaptosomes. J. Biol. Chem. 285, 8639-8645 (2010).

18 Silveira, P. C., Streck, E. L. \& Pinho, R. A. Evaluation of mitochondrial respiratory chain activity in wound healing by low-level laser therapy. J. Photochem. Photobiol. B 86, 279-282 (2007). 